\title{
The Use of Educational Media in Endoscopy Training
}

\author{
Mohammad Fawad Khattak
}

Funding: The author(s) received no specific funding for this work.

Potential competing interests: The author(s) declared that no potential competing interests exist.

\begin{abstract}
When delivering or planning any learning intervention it is important to consider the available educational media. Educational media can be used to supplement, consolidate or be the basis of any learning intervention and they can come and be delivered in a variety of different formats. In this article we look at the use of educational media in endoscopy training.
\end{abstract}

\section{Introduction}

When delivering or planning any learning intervention it is important to consider the available educational media. Educational media can be used to supplement, consolidate or be the basis of any learning intervention and they can come and be delivered in a variety of different formats including (Bates, 2015);

1) Face-to-face teaching

2) Audio e.g podcasts

3) Video e.g Youtube

4) Graphical

5) Simulation/Virtual Reality (VR)

6) Text/Powerpoint Presentations

\section{Simulation}

Traditionally the acquisition of skills by a physician was done via a "see one, do one, teach one" approach. This apprentice-style of skill acquisition is no longer considered to be a safe or acceptable method of learning due to concerns regarding potential care and patient safety and this has led to a change in our practises for teaching and learning skills, with the introduction of simulation training being one of the key changes.

Simulation training involves creation of a situation or environment that allows participants to experience a representation of a real event for the purpose of practice, learning, evaluation, testing, or to gain an understanding of systems or human actions (Battista and Nestel, 2019).

Simulation is particularly useful in allowing novices to learn or practise a skill in a low risk environment 
with no concerns about patient safety (Khan et al., 2018). Simulation training allows experts to give instant feedback and can be used as a requirement prior to allowing students/doctors to do a procedure/skill to ensure a basic level of competency has been achieved prior to any real life use (Thorne et al., 2020). Although simulation training is a relatively new concept in medical education it has been extensively used in other high-risk professions such as aviation to much benefit (Al-Elq, 2010).

As a gastroenterology trainee one of the main aims during training is to become competent in a number of different endoscopic procedures. Endoscopy is a safe procedure in expert hands however risks still exist and these are exacerbated when a novice is performing the procedure. Endoscopy simulators such as the one demonstrated in Figure 1 have been introduced to train beginner endoscopists on the basics of scope handling and tip control, so that once adequately trained they are able to do the procedures on actual patients with far greater success and with less risk to the patient.

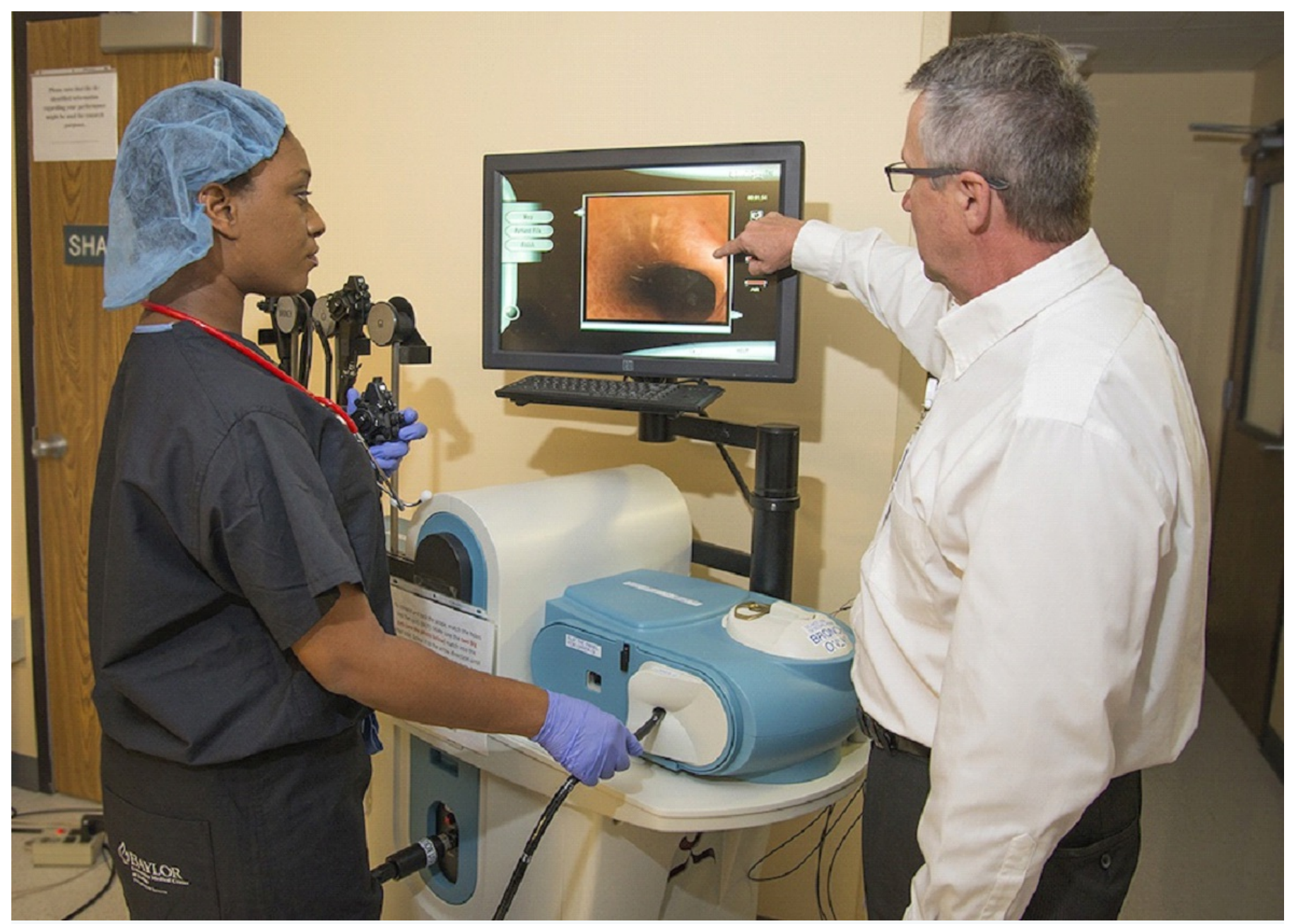

Figure 1:- Example of an endoscopy simulator (Healthy Simulation , 2020)

\section{Joint Advisory Group on GI Endoscopy (JAG) Upper Gastrointestinal (UGI) Mandatory Course}

As part of formal upper Gl endoscopy training all endoscopists in the United Kingdom must take part in a 
three day JAG course. This is intended to be at the start of the endoscopist learning process and involves a intense three day 50:50 split between simulator training followed by endoscopy on real patients under expert supervision (Siau et al., 2018).

The aim of this three day course is to:-

1) Teach the basic theoretical knowledge of using endoscopy to investigate the Upper GI tract; how an endoscope works, relevant anatomy, indications, risks/benefits etc.

2) Acquire basic scope handling skills and tip control by practising multiple example cases on both a low fidelity plastic mannequin simulator and a high fidelity computer simulator

3) Perform upper Gl endoscopy on patients under expert supervision

All three aims of the course involve the use of a significant amount of media.

The first aim is addressed mainly through lectures and Powerpoint presentations containing both text, graphical and video media. When teaching a small group (5-10 participants) theoretical knowledge with no significant higher level analysis required traditional didactic teaching using presentations is appropriate to enable the main outcome which is simply delivery of information from an expert to a group of students complemented by the use of pictures and video to help orientate the students prior to them using the simulator. This process does this without using significant resources in terms of time and personal and alternative methods for this section such as problem based learning are unlikely to increase the effectiveness of learning significantly whilst significantly increasing the amount of resources used (Long and Lock, 2014).

The second aim is addressed by supervised endoscopy on the simulators. The simulator has a real life endoscope which when inserted into the computer behaves like an actual endoscope and the computer generates a three-dimensional anatomically accurate upper GI tract. Multiple different normal and abnormal cases are available in order to pose different challenges to learners. This allows learners to practise the procedure without worries about patient safety and allows students to repetitively practise the procedure a far greater amount in a much shorter time period than would have otherwise been possible if done on real patients (Powell-Laney, 2010). Experts are able to give real time feedback whilst performing the procedure on the simulator and the simulator itself is able to give both qualitative and quantitative feedback. Studies have compared the effectiveness of simulation training in the JAG course and demonstrated that participants in courses containing simulation training had higher confidence in all technical skills surveyed, with follow up showing those who used simulation based teaching achieving competencies earlier than their colleagues (Siau al., 2020).

After the first two aims are achieved trainees then perform upper Gl endoscopy on patients under supervision. Initially prior to the procedure trainees will spend 5 minutes with the supervisor to go through the learning objectives and plan for the procedure and following the procedure a Direct Observation of Procedural Skills (DOPS) form is done as part of formative feedback. 
The JAG course is a resource heavy course requiring numerous days, many trained supervisors, numerous endoscopy rooms, endoscopy nurses, patients and expensive simulator equipment. It nicely uses a wide variety of educational media at different stages, recognising that different media is more appropriately used in different scenarios and that they help the course achieve its learning outcomes in different ways (Singh, Sedlack and Cook, 2014).

Simulation training is a validated way to help trainees achieve competency in endoscopy with numerous studies showing that they reduce the learning curve and subsequently the time required for trainees to achieve competency (Siau al., 2020) (Cohen et al., 2004).

\section{Conclusion}

Educational media are tools that can be used in a variety of ways and when analysing their usefulness it is important to recognise that the effectiveness of the media will be dependent on how they are used and the scenario or situation that they are used in.

Simulation based training will increasingly be used in medical education as technology develops resulting in higher fidelity and more immersive experiences which can accurately replicate real life procedural skills without any patient safety concerns (So, et al, 2019). As virtual reality (VR) technology develops simulation training in the future will be able to accurately replicate a wide variety of medical and surgical procedures and this is area which will likely revolutionise medical education.

Currently simulator based training is expensive and for certain procedures current limitations in technology result in reduced accuracy and effectiveness of simulation (Sawyer and Gray, 2016).

Simulation should ideally always be a complement to real world learning as simulation is unlikely to create the atmosphere or pressures that one faces when having to do an emergency procedure.

In summary simulation based training has many benefits when used in the teaching of endoscopic procedural skill acquisition and studies have demonstrated that it leads to earlier competency and more confidence for trainees with the future of simulation and VR showing much promise.

\section{References}

Al-Elq, A. (2010). Simulation-based medical teaching and learning. Journal of Family and Community Medicine, [online] 17(1), p.35. Available at: https://www.ncbi.nlm.nih.gov/pmc/articles/PMC3195067/ [Accessed 15 March 2021].

Bates, T. (2015). Teaching in a digital age : guidelines for designing teaching and learning. Burnaby, British Columbia: Sfu Document Solutions, Simon Fraser University.

Battista, A., Nestel, D. (2019). Simualtion in Medical Education. In: Swanwick T, Forrest, K., O'Brien, B.C. (ed). Understanding medical education: evidence, theory and practice. Oxford: John Wiley \& Sons, Ltd, p.151-159. 
Cohen, J., Cohen, S., Vora, K.C., Burdick, J.Steven., Hawes, R.H. and Xue, X. (2004). Impact of Virtual Reality Simulator Training on the Acquisition of Competency in Colonoscopy: Final Results of Multi-Center Randomized Controlled Trial. Gastrointestinal Endoscopy, 59(5), p.P110.

HealthySimulation.com. (2020). Endoscopy Simulator Healthcare Simulation Available at: <https://www.healthysimulation.com/endoscopy-simulator/>; [Accessed 15 March 2021].

Khan, R., Plahouras, J., Johnston, B.C., Scaffidi, M.A., Grover, S.C. and Walsh, C.M. (2019). Virtual reality simulation training in endoscopy: a Cochrane review and meta-analysis. Endoscopy, 51(07), pp.653-664. Long A., Lock B. (2014). Lectures and large groups. In understanding Medical Education: Evidence, Theory and practice (Ed) by Tim Swanwick. Wiley Blackwell, London, UK, p142.

Powell-Laney, S. K. (2010). The use of human patient simulators to enhance the clinical decision making of nursing students. Available at: https://scholarworks.waldenu.edu/cgi/viewcontent.cgi? article $=1016 \&$ context $=$ dilley (Accessed 15 March 2021) Siau, K., Green, J.T., Hawkes, N.D., Broughton, R., Feeney, M., Dunckley, P., Barton, J.R., Stebbing, J. and Thomas-Gibson, S. (2018). Impact of the Joint Advisory Group on Gastrointestinal Endoscopy (JAG) on endoscopy services in the UK and beyond. Frontline Gastroenterology, 10(2), pp.93-106.

Siau, K., Hodson, J., Neville, P., Turner, J., Beale, A., Green, S., Murugananthan, A., Dunckley, P. and Hawkes, N.D. (2020). Impact of a simulation-based induction programme in gastroscopy on trainee outcomes and learning curves. World Journal of Gastrointestinal Endoscopy, 12(3), pp.98-110. Singh, S., Sedlack, R. and Cook, D. (2014). Effects of Simulation-Based Training in Gastrointestinal Endoscopy: A Systematic Review and Meta-analysis. Clinical Gastroenterology and Hepatology, 12(10), pp.1611-1623.e4.

So, H.Y., Chen, P.P., Wong, G.K.C., Chan, T.T.N. (2019). Simulation in medical education; JRCPE; 49, p. 5257.

Swayer, T., Gray, M.M. (2016). 'Procedural training and assessment of competency utilizing simulation' Semin Perinatol; 40: 438-446

Thorne, C.J., Kimani, P.K., Hampshire, S., Begum-Ali, S. and Perkins, G.D. (2020). Feedback in advanced life support: A quality improvement initiative. Resuscitation, 155, pp.189-198. 
